modèles …!.?!

linguistiques "थं:

\section{Modèles linguistiques}

$66 \mid 2012$

Langues et cultures régionales de France : Béarn et Gascogne (I)

\title{
Essai de toponymie non romane de la plaine de Nay (Béarn)
}

Joseph Rebenne

\section{OpenEdition}

\section{Journals}

Édition électronique

URL : https://journals.openedition.org/ml/308

DOI : $10.4000 / \mathrm{ml} .308$

ISSN : 2274-0511

Éditeur

Association Modèles linguistiques

Édition imprimée

Date de publication : 1 décembre 2012

Pagination : 111-135

\section{Référence électronique}

Joseph Rebenne, "Essai de toponymie non romane de la plaine de Nay (Béarn) », Modèles linquistiques

[En ligne], 66 | 2012, mis en ligne le 05 mars 2013, consulté le 01 juillet 2021. URL : http://

journals.openedition.org/ml/308; DOI : https://doi.org/10.4000/ml.308 


\section{Essai de toponymie non romane de la plaine de Nay (Béarn)}

\section{Joseph Rebenne}

\section{Avertissement}

Le présent document provient d'un travail bien plus conséquent. Les faits qui y sont examinés, et que nous ne pouvons exposer ici, nous ont engagé à faire l'hypothèse d'une origine proto-basque et basque de notre région. Il ne s'agit donc pas d'un présupposé gratuit. Bien entendu, il y a place pour le débat, mais celui-ci doit voir s'affronter des faits et non des convictions.

Par ailleurs, Michel Grosclaude ayant réalisé il y a quelques années un remarquable Dictionnaire toponymique des communes de Béarn (voir Bibliographie), notre propos n'est pas de nous y substituer. Mais Michel Grosclaude, qui était d'Orthez, ne pouvait connaître dans le détail les subtilités du relief de la plaine de Nay comme peut les connaître un natif. Par ailleurs, son étude concernait les quelque 480 villages béarnais, exercice autrement difficile que celui qui consiste à examiner l'origine d'une petite vingtaine de toponymes.

Situation de la plaine de Nay

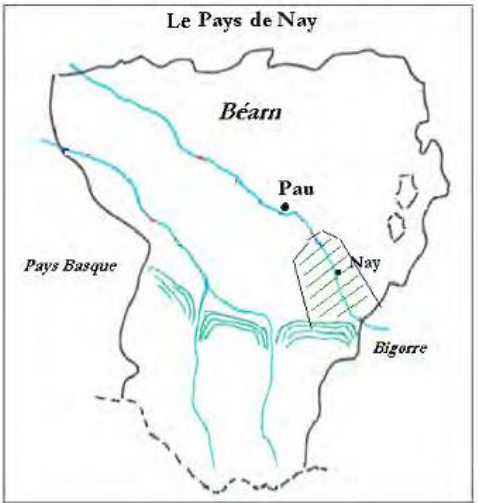

Fig. 2

Situation du Pays de Nay en Béarn

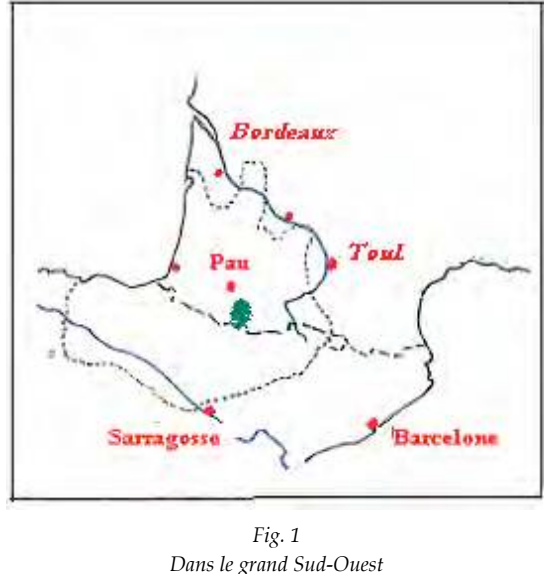




\section{I - Les difficultés de la toponymie}

L'étymologie est la science des ânes, selon un linguiste du 19e siècle. Que dire alors de la toponymie, où même les plus grands, malgré leur prudence, ont multiplié les erreurs ? C'est que, dans ce domaine, on trouve souvent ce que l'on cherche, dans la direction où l'on cherche. Prenons le cas d'Idron, village béarnais de la région paloise. Celui qui ne voit derrière les noms de nos bourgs que l'origine, latine ou germanique, de noms d'hommes, trouvera la solution dans Iteraeus, nom latin. Celui qui cherche du côté proto-basque, la verra plutôt dans Itur-une, la « source ». Le recensement de Gaston Fébus en 1385 va départager les deux interprétations, car il révèle l'existence, en limite d'Idron, d'un hameau nommé Hondarede, la « source froide », variante romane d'Iturun.

C'est dire qu'il convient d'aborder la toponymie avec modestie, protégés du pire, espérons-le, par le respect de quelques principes.

\section{I) Ne pas sous-estimer l'imprégnation aquitaine}

Les Pyrénées-Atlantiques ne possèdent ni épigraphie ni monuments romains. Le développement de Beneharnum [Lescar, ndlr] et d'Iluro [Oloron, ndlr] fut tardif et modeste, et la colonisation agricole latine directe s'arrêta à la limite du plateau de Ger. Certes, les indigènes aquitano-romains ne tarderont pas à se parer de noms romains, mais cette terre, qui parlait aquitain et qui parlera basque jusqu'au Moyen-Âge, a de fortes chances d'avoir nommé les créations indigènes selon ses propres coutumes, réservant les noms d'homme pour les sites des grandes exploitations agricoles du premier millénaire.

\section{II) Se montrer prudent quant aux témoignages écrits}

Ceux-ci peuvent se révéler précieux, mais ils ne sont pas fiables, du moins dans les textes latins médiévaux. Obligés de traduire les noms de village ou, à tout le moins, de leur donner une forme latine, les clercs verseront souvent, hélas, dans l'étymologie populaire. C'est ainsi que Coarraze est attesté sous la forme Cauda rasa, la « queue coupée ». Dans d'autres cas, on peut s'interroger : la traduction d'Escoubès par Scoparius révèle-t-elle un original Escober ou une étymologie populaire, escoubès, qui signifie en béarnais « fabricants de balais »?

D'autre part, la graphie est une convention dont la lecture, dans les textes anciens, n'est pas assurée. Comment lire Faget ? Haget, Hayet, Fayet ? Il est plus que probable que le $f$ doit se prononcer $h$; les toponymes basques voient en effet à cette époque nombre de leurs $h$, pourtant incontestables, écrits $f$. Quant au $l$ de Belste (Beuste), est-il une hyper 
correction erronée ou trahit-il un état de langue où les $l$ devant consonne ne sont pas encore vocalisés?

Quoi qu'il en soit, on ne pourra aucunement faire l'impasse sur la consultation des premières formes écrites, qui donnent parfois de précieuses indications. Lusson par exemple est attesté sous la forme Luysson en 1385, dans laquelle on peut voir lohitzun, le « lieu marécageux ». Cette dernière pourrait fort bien être la forme étymologique, à condition expresse de vérifier - ce qui n'a pour l'instant pas été fait - que le territoire de ce village était effectivement marécageux.

\section{III) Examiner très attentivement les sites}

Un grand nombre de nos villages ayant hérité du nom du lieu-dit sur lequel ils s'installaient, l'examen très attentif des sites sera obligatoire quand bien même, en première analyse, un anthroponyme serait convaincant. Prenons en exemple le cas de Livron qui se prononce en béarnais [liu'rũ]. Une origine anthroponymique "Liberius/one » qui donnerait [li ${ }^{\mathrm{u}^{\prime}}$ rjũ $]$ est acceptable. Mais le basque "Loirune »

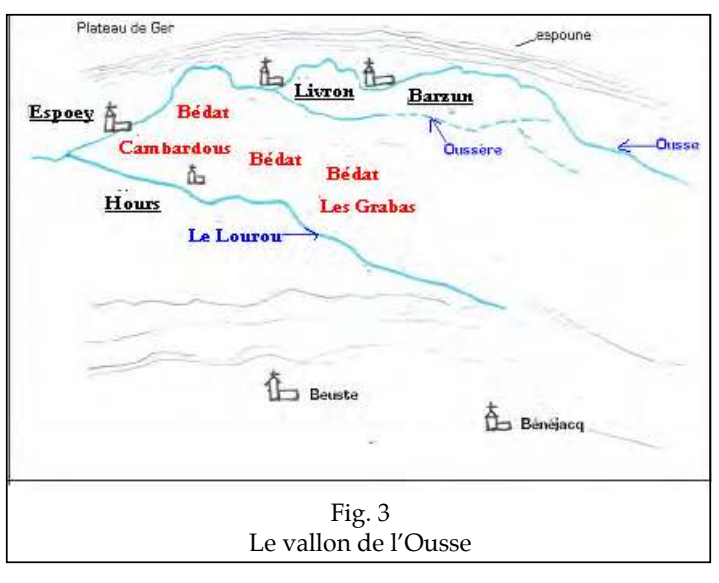

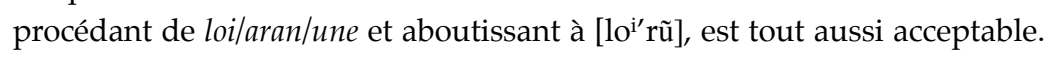
Dans ce dernier cas le site doit parfaitement correspondre, loirune signifiant « le val marécageux » Or le vallon de l'Ousse, au niveau du village, est appelé Les Grabas (les terrains bourbeux), trahissant une origine marécageuse. Je suis donc pour ma part d'autant mieux convaincu que le toponyme premier est Loiru, que trois «Bedats » entourant le village rappellent les anciens bois en défens destinés à favoriser le drainage de l'eau, que l'espace entre Hours et Espoey est appelé « Cambardous » c'està-dire champ boueux et que coule à proximité un ruisseau, le Lourou, dont le nom est d'évidence le même.

La concordance phonétique avec une hypothèse n'est donc pas une preuve, loin de là. C'est l'examen attentif du site du hameau primitif qui sera déterminant. Ainsi la comparaison des sites de Pardies, Baliros, Boeil et Lassun avec ceux de Pardies-Monein, Balirac, Boeil-Boeillo et Laas se révèlera très parlante. 


\section{IV) Tenir compte de l'évolution phonétique des langues}

Imaginons un toponyme aquitain du IVe siècle tel que Bukele. Il a toutes les chances de se retrouver aujourd'hui sous la forme Boulh, Boeil en zone romanophone, et sous la forme Mouguère en zone bascophone. Il s'agit certes d'un cas limite, auquel on peut opposer de nombreux exemples de stabilité, mais, face à un toponyme, cela nous met dans l'obligation de nous interroger, selon la situation linguistique, sur ses antécédents possibles.

On prend donc de grands risques à imaginer que des formes analogues puissent avoir la même origine en zone aquitaine et en zone celte. On doit se garder de généraliser certains cas troublants tels que l'hydronyme Lez qui pullule en Aquitaine et que l'on retrouve de l'autre côté du Rhône (cf. la Lèze dans le Comtat Venaissin). Certes, les évolutions celtes, latines et aquitaines ne recouvrent pas à elles trois l'ensemble du spectre phonétique, et un mot comme Lez a pu ne subir aucune mutation dans ces trois langues. Mais ce ne peut être le cas général.

En conséquence, pour aborder cette recherche toponymique, la connaissance d'un minimun de racines du basque médiéval ne suffit pas. Il faut y ajouter celle des mutations qui modifieront la morphologie de la langue romane proto-gasconne entre les invasions barbares et l'empire de Charlemagne. Celles qui nous seront utiles ici sont les suivantes :

- Disparition du - $n$ - intervocalique :

luna $\Longrightarrow$ lua

- Réduction de -nd- à -n- : grande $(\mathrm{m}) \Longrightarrow$ grane

— Réduction des groupes -rs-, -rts-, à -ss- :

ursus $\Longrightarrow$ os

— Vocalisation de $-l$ en finale de syllabe :

caldu $\Longrightarrow$ caut

- Le basque - $t z$ - donne -st-

Il va s'agir pour nous de remonter de la forme actuelle béarnaise vers une possible forme basque ou proto-basque en tenant compte que ces mutations ont pu jouer. On peut ainsi, à partir d'Assat, reconstituer comme original possible: Assat, mais aussi Assanate, Artsate ou Artsanate.

Notons enfin qu'un toponyme basque commençant par une voyelle suivie d'une seule consonne perd souvent sa voyelle initiale lors de son adaptation au roman. Nous avons les exemples d'Ataraxe devenu Tardets et en Espagne de Ibarrangelua romanisé en Varanguela. Ce trait paraît très courant dans les doubles séries des toponymes béarnais basco-aquitains.

\section{V) Travailler sur la prononciation béarnaise du toponyme.}

Nos différents toponymes ont été orthographiés dans la graphie béarnaise médiévale. Ils sont aujourd'hui lus à la française, lecture qui les 
dénature artificiellement. C'est ainsi que la prononciation authentique de St Faust est [sen'ha ${ }^{u} \mathrm{~s}$ ], celle de Baudreix [ba $\left.{ }^{{ }^{\prime}} \mathrm{dr} \varepsilon f\right]$, etc. C'est donc de cette forme béarnaise qu'il faut partir pour essayer de retrouver l'étymologie du nom.

\section{VI) Se méfier des convergences de formes}

Se pénétrer de l'idée qu'il est fort peu probable qu'un mot ait un même sens à mille kilomètres de distance. Se méfier des convergences de forme qui peuvent recouvrir des étymologies différentes et se convaincre que ce n'est pas parce qu'un nom correspond parfaitement, phonétiquement, à un mot latin que là est son origine.

\section{L'aire culturelle du Pays de Nay}

> Le Pays de Nay appartenait à l'aire de parcours des pasteurs nomades montagnards proto-basques, c'est-à-dire à leur aire économique et culturelle.

> Nous ne sommes pas dans une zone de culture indo-européenne mais euskarienne. Les villages ne portent pas ici le nom d'un hypothétique fondateur, mais celui du lieu où s'établissent les hameaux. La seule exception à ce fait sera due à la conquête romaine.

> Les autochtones nommaient les sites qui, plus tard, accueilleront les villages en fonction de leurs préoccupations pastorales. Importaient pour eux, au cours de leur transhumance, notamment :

— les singularités du relief, ses difficultés (marécages, talus, etc.)

— les pâturages et leur catégorie : prairies humides, landes, etc.

— les passages obligés : gués, points d'eau, etc.

Et, bien entendu, la présence éventuelle de telle villa aquitano-romaine.

Sur ces points, leur langue était riche et il nous est difficile d'en saisir les nuances :

- Buka, bal, représentaient les hauts et longs talus formant barrière. Cf. le basque moderne : muga, bar, signifiant «talus, limite, frontière ».

- Ana était la nourriture pour le bétail, anda, ala, eunste les prairies et pâturages, larr la lande.

- Ibi signifiait le « gué », ithur la « source », carr/harr la «pierre », lohi le «marécage », artzaina le « berger », ate le " passage » etc....

- les singularités étaient décrites par analogie avec leur environnement : la sinusoïde par adar, la " corne », et tel type d'éperon par ira, la « fougère ». 
Les suffixes étaient d'une grande richesse :

- locatifs : -eta, -ate, -aga,-oz, -gune, -une (attesté également au Pays Basque : $u, u e, u n)$.

Exemple : dans «latz-une», le suffixe -une- précise qu'il s'agit du « lieu du ruisseau $\gg($ latz)

- quantitatifs : $-z u,-t z u,-t z$, etc.

Exemple : Leiatzun, le lieu (un) où se trouve beaucoup $(t z)$ de sable (leia).

Mais ces suffixes ne sont pas utilisés partout et à la même époque. Ainsi en Béarn -une semble privilégié à une époque tardive, alors que $-o z$ l'était lors de la conquête.

Pas d'humour, pas de jeu intellectuel, un chat est appelé un chat. À partir du $11^{\text {e }}$ siècle, lors des premiers défrichements du saltus d'Asson, Capbis (« la source du Beez ») et Mifaget (« au milieu de la hêtraie ») apparaissent comme des traductions.

\section{III - Les doubles séries de toponymes en zone de diglossie}

La région a été peuplée de temps immémorial d'Aquitains et de Vascons, c'est-àdire de proto-basques. C'est du moins l'hypothèse retenue, compte tenu de faits étudiés par ailleurs, et que nous ne pouvons bien entendu passer ici en revue. Près de mille ans de diglossie ont suivi l'implantation du latin/ roman. Il est donc normal de trouver dans la région de Nay des toponymes basco-aquitains, des toponymes romans, et un ou autre toponyme sur nom d'homme latin (adopté par les aquitano-romains), et probablement vain de chercher ailleurs.

Mais si le Pays de Nay a parlé basque en des temps révolus, cependant pas si lointains, on pourra légitimement s'étonner de ne pas y trouver de toponyme qui sonne basque. La réponse à cette objection ne pose pas de gros problème. Une promenade en Soule, province basque s'il en est, y suffit.

Nous y observons en effet que les noms de localités, les noms officiels s'entend, semblent on ne peut plus gascons. Tardets, Laruns, Aussurucq, Sauguis, Larrau, Moncayole, Ordiarp, Arros, etc. ne dépareraient absolument pas en Béarn. Et pour cause : ces noms sont effectivement gascons. Ils proviennent d'un original proto-basque modifié au cours des siècles par la phonétique du gascon. Ce sont eux, et non leur variante basque, qui seront retenus dans la nomenclature officielle, car l'écrit était gascon et non basque.

Aujourd'hui nous pouvons, grâce à l'annuaire téléphonique, comparer ces toponymes avec leur doublet souletin : Ataraxe, Lahuntze, Altzürükü, Zalgize, Larraine, Mitikile, Urdinarbe, Arroze, etc....Ceux-ci proviennent du 
même original proto-basque que les précédents, mais modifié cette fois-ci, par la phonétique basque. Car le basque, comme toute langue, évolue et se modifie. Si celui-ci avait disparu de Soule il y a deux ou trois siècles, et cela a failli être le cas, nous ignorerions tout de la série basque disparue avec cette langue.

En ce qui concerne les micro toponymes, bien plus volatils que les toponymes, car ils sont pratiqués très localement, ils auraient été en grande partie éliminés, remplacés par des appellations béarnaises, à l'exception des noms de maisons et de quelques autres bien installés.

Je relève en effet une trentaine de noms de quartiers dans mon village de Pardies, dont une vingtaine de langue romane, et, tout de même, une dizaine de type basque, comme par exemple l'Arrondade (larrondate, « le fond de la lande »), Carrique (karrika, « le chemin »), Carobe (garrobi, « le val des pierres, le lit du torrent»), Cerbou (serraburu, " l'avancée de la colline »), Bérétardou (bere-t-ardu, « le lieu en bas des vignes »), le Luz (lohitzu, « le ruisseau marécageux »), l'Escourre (peut-être eska-urra, « le canal », qui se dit ur-aska en basque), la Mugue (" le talus limite ») qui sépare Pardies de St Abit, etc. Tous ces micro toponymes correspondent parfaitement au site. En ce temps de transition linguistique, combien auront survécu dans vingt cinq ans, parmi les premiers comme parmi les seconds ? Dix pour cent au mieux. Signalons encore, sans nous éloigner beaucoup, le bois de Berot à Assat, situé plein sud dans un cirque protégé et remarquable par sa douceur les jours d'hiver, pour peu que le soleil brille. L'étymon bero, "chaud ", est ici plus que probable. Si nous cherchions dans nos villages auprès des anciens connaissant encore la micro-toponymie des lieux, nous serions peut-être surpris par la récolte. En zone de diglossie nous avons donc deux séries de toponymes, une pour chaque langue.

Les noms de village non romans de la région de Nay appartiennent à la série gasconne, la série basque ayant disparu sans laisser de trace lors de la débasquisation de la région, à l'exception de Pardies comme nous le verrons. Il va donc nous falloir, pour en trouver l'origine étymologique, reconstituer la série atavique.

Nous avons au total trente villages et hameaux :

\section{A. 21 antérieurs au $11^{\mathrm{e}}$ siècle :}

- dont 2 sur nom d'homme latin : Claracq et Bénéjacq et :

17 d'origine supposée aquitaine ou basque :

Assat, Pardies, Baliros

Arros, Nay, Angaïs 
Beuste, Lagos, Coarraze

Lassun, Arthèz, Guataram

Laber, Igon, Asson

Boeil, Baudreix

> et 2 d'origine incertaine mais peut-être bien aquitaine :

(Saint), Abit

Mirpeix

Laber et Lassun étaient les noms anciens de Lestelle et de Montaut, bastides créées au $14^{\mathrm{e}}$ siècle à proximité de ces deux hameaux qu'elles ont absorbé. Guataram est le nom ancien de Betharram jusqu'au $17^{\text {e }}$ siècle. $C^{\prime}$ est ce nom ancien que nous gardons ici. Il ne faudrait pas imaginer par ailleurs que les 17 villages basco-aquitains étaient en place lors de la conquête romaine. Ils étaient en place en l'an mil, ainsi que Clarac et Bénéjacq, qui datent probablement des $3^{e}$ et $4^{\mathrm{e}}$ siècles. Remarquons que ces toponymes sont tous situés dans la vallée du gave ou ses abords immédiats (Asson).

\section{B. 7 de création médiévale}

portant des noms romano-béarnais, l'original basque n'ayant pas survécu, s'il existait, car, à cette époque, seul le romano-béarnais était langue officielle :

Capbis, Mifaget,

Bezing, Bruges

Bordes, Bourdettes, Bordères

Dans les coteaux de colonisation médiévale, tous les villages portent un nom roman : Mifaget, Capbis, Lys, etc. Au tournant probablement de l'an Mil, la pression grandissante du gascon aidant, les créations récentes seront, non plus adaptées phonétiquement parlant au roman, comme précédemment, mais traduites dans celui-ci. Mifaget, par exemple, a très vraisemblablement été précédé par *Bagarte. Le contraire serait surprenant car nous rencontrons encore dans cette zone des micro-toponymes d'étymologie basque, alors que les micro-toponymes sont plus volatils que les noms de village, étant moins pratiqués et non traduits. Citons par exemple: Le bois de Laspar (latz-pare, " les deux ruisseaux »), entre Mifaget et Pedestarres, petite éminence boisée circulaire dans laquelle prennent effectivement naissance deux rus, ainsi que le Landistou (l'anditzu, « le ruisseau des prairies ») qui coule dans le « plat » de Lys au milieu des prairies. 


\section{2 de création tardive, fin $19^{\mathrm{e}}$ siècle :}

\section{Le Haut de Bosdarros}

\section{Saint Vincent}

Ces deux villages possèdent également un nom béarnais, La Chapelote pour le premier et Sen Bincens pour le second, mais ils vont disparaître avec la pratique de la langue béarnaise, de la même façon que disparurent probablement les originaux basques de Capbis, Mifaget, car désormais ce n'est plus le béarnais qui est la langue officielle, mais le français.

Ce sont les toponymes de la série proto-basque et basque auxquels nous allons nous intéresser maintenant. Il nous faut au préalable jeter un coup d'œil sur les principales caractéristiques physiques de la région de Nay, car celles-ci ont de fortes chances d'avoir présidé à la dénomination des sites.

\section{IV - Géographie du Pays de Nay}

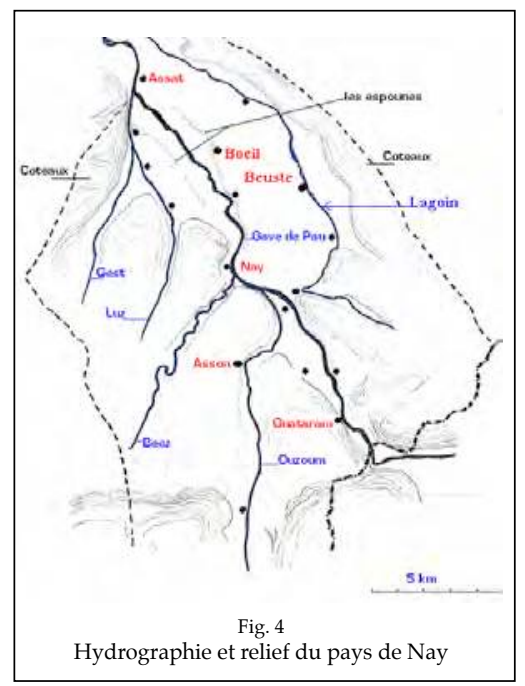

Le Pays de Nay correspond aux deux cantons de Nay-Est et de NayOuest. Il comprend :

- L'arribère (ou vallée fluviale) du gave de Pau entre Betharram d'une part, et Assat d'autre part.

- L'extrémité Est des coteaux d'entre deux gaves qui seront défrichés au Moyen-Age. Cette partie du saltus correspond aux bassins fluviaux de l'Ouzoum, du Beez, du Luz et du Gest.

Cet ensemble s'appuie au sud-est sur la Bigorre, au sud sur le massif montagneux. Il est délimité à l'Est

l'Ouest ne correspond à aucune singularité. par le chemin H IV. La délimitation à

A l'entrée en Béarn, le lit du gave, encaissé jusqu'à Montaut et Bétharram, s'élargit brusquement pour se resserrer de nouveau quelques kilomètres plus loin à Igon, au-delà duquel il s'ouvre très largement jusqu'au relatif resserrement d'Assat.

De part et d'autre du gave nous trouvons :

- sur la terrasse inférieure une zone de saligues et de prairies.

- à la jonction terrasse inférieure/terrasse supérieure un talus abrupt de $10 \mathrm{~m}$ que j'appellerai ici par son nom béarnais d" « espoune ». 
- sur la terrasse supérieure une vaste plaine consacrée aujourd'hui aux cultures, mais anciennement lande pastorale dépourvue de point d'eau.

- puis, rive droite, assez loin du pied de la colline, un ruisseau, le Lagoin, et, rive gauche, deux autres ruisseaux collés aux coteaux, le Luz et le Gest.

— rive droite, entre la colline et le Lagoin, une assez vaste zone de prairies humides, réceptacle naturel des eaux de ruissellement des coteaux.

- enfin, de part et d'autre, deux lignes de coteaux dominant l'ensemble d'une centaine de mètres.

Les singularités de cette arribère fort utiles pour notre analyse sont les suivantes :

- Les talus abrupts des deux espounes qui encadrent à distance le lit du gave, le dominant d'une dizaine de mètres.

- Les zones de barthes et de prairies humides situées entre les ruisseaux et les coteaux.

- Les promontoires rocheux situés à Coarraze et au nord de Nay.

- Le resserrement fermant la vallée au sud, au contact de la Bigorre.

- Le chemin de transhumance (la bie aussalese) suivant la ligne haute des coteaux de l'ouest et venant traverser le gave au gué d'Assat.

\section{V - Analyse des toponymes basco-aquitains du Pays de Nay}

\section{AssAT}

Prononciation béarnaise : [as'sat]

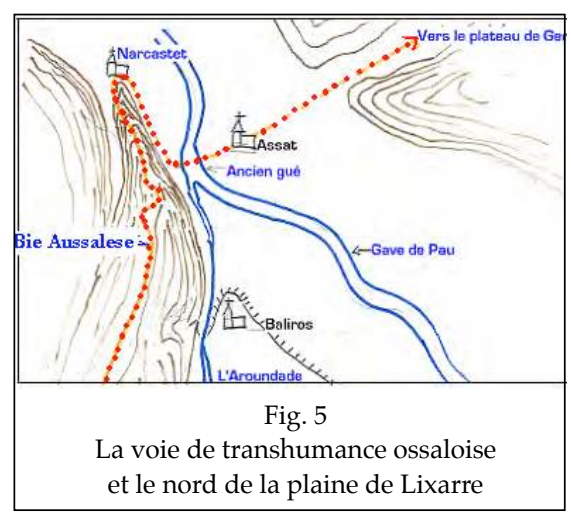

Nous avons ici deux singularités :

- La première est que le gave vient lécher la falaise des coteaux au regard du village, ménageant un étroit passage et obligeant la route rive gauche à emprunter quelque peu la colline jusqu'aux approches de Baliros.

- La seconde est que la Bie

transhumance ossalois vers le plateau de Ger, traversait le gave sur l'ancien gué, à proximité du pont actuel, après être descendue des crêtes au niveau de Narcastet où nous la trouvons endimanchée sous 
la forme de "Viossalèse ». Au début du second millénaire un fort, ancêtre du château actuel, sera construit à proximité du gué afin de contrôler les montagnards qui n'étaient pas des tendres. Ceux-ci d'ailleurs, qui nécessitaient sur leur parcours des pâturages pour leurs troupeaux, empêchaient l'établissement de hameaux à proximité de leurs pâtures de transhumance. Ce fait explique peutêtre qu'à l'époque les villages les plus proches du gué, Boeil, Beuste et Angaïs, se trouvaient à plusieurs kilomètres de là, réservant une très vaste lande au pâturage des troupeaux transhumants. Le fort ne suffisait probablement pas car, en 1280, une bastide sera édifiée près du pont actuel, dans le but, on peut l'imaginer, de réduire les prétentions et les exactions ossaloises.

\section{Première hypothèse}

Assat proviendrait d'Artzate, tout comme Ossès provient d'Ortzaitze. Or artzalate, c'est le «passage (vers le pays) des ours». Ceux-ci pullulaient dans le saltus d'Asson et l'on en trouvait certainement dans les coteaux bordant la plaine de Licharre. Ate, le passage, existe bien ici.

Deuxième hypothèse

Assat proviendrait d'artzan/ate, le «passage des bergers ». Le site correspond parfaitement à l'étymologie : artzanate $\longrightarrow$ arsaate $\longrightarrow$ assat conformément à la phonétique du gascon. Artzana serait ici une variante d'artzaina, " berger », qui doit s'interpréter ardi-zana, le " soigneur de brebis ». Préférence pour la seconde hypothèse :

artzan/ate, le « passage des bergers ».

\section{$>$ ANGAIIS}

Prononciation béarnaise : [an'gais]

Nous venons de voir qu'une vaste lande occupait l'espace entre Beuste, Boeil et Assat. Le village qui s'y établira, à distance prudente du gué, portera son nom. On peut interpréter celui-ci en effet comme :

Anda-Gaitz, « la vaste prairie » ou bien :

Ana-Gaitz, «l'importante (ou la mauvaise) nourriture pour le bétail ».

\section{PARDIES}

Prononciation béarnaise : [par'dîjj]

Le point de vue de Michel Grosclaude, si convaincant et argumenté soit-il, n'a pas résisté à l'analyse comparative des sites de Pardies-Piétat et de Pardies-Monein.

Contrairement à ses voisins Baliros, St-Abit et Arros, le village n'est pas au pied des coteaux mais à mi-chemin entre l'espoune et ces derniers, 
face à la trouée du Gest, se tenant prudemment à distance des deux ruisseaux (le Luz et le Gest) qui se rejoignent peu après et inondent régulièrement l'espace compris entre son bâti et les collines (Luz $<$ lohitz, marais). Cet espace est d'ailleurs occupé par un bois en défens, le Bédat, destiné, comme nous l'avons vu plus haut, à accélérer le drainage des « aygats ». Le drame qui durant l'été 2007 a endeuillé le village témoigne de cette réalité.

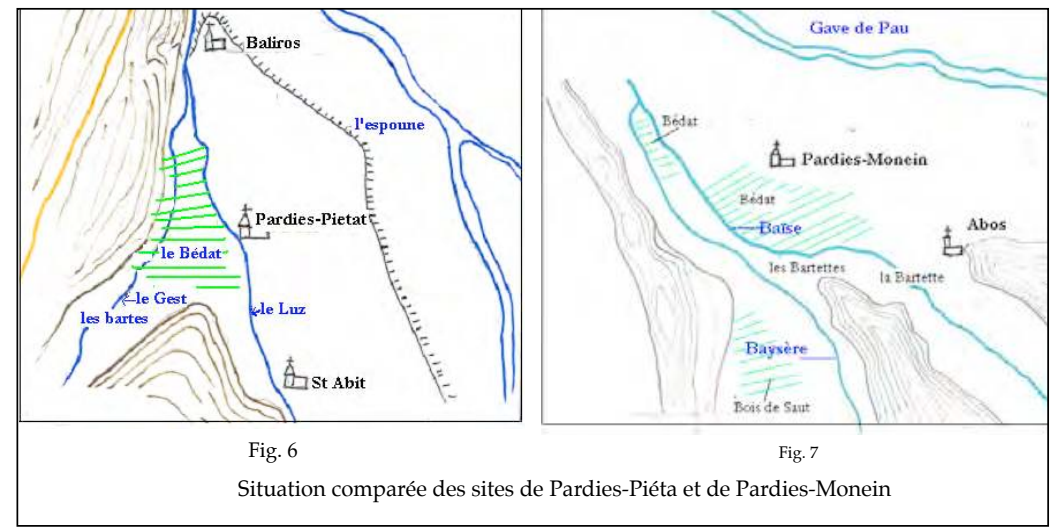

Or le site de Pardies-Monein est identique, à mi-chemin entre les coteaux et le gave, face à la trouée de la Baïse et de la Baysère qui se rejoignent à proximité. L'espace compris entre le village et les coteaux est, comme à Pardies-Pietat, occupé par des barthes et une forêt en défens.

La Baïse évacue les eaux pluviales d'un bassin non négligeable commençant sur les hauteurs de Lasseubetat et comprenant les communes de Lasseube, Lacommande et Parbayse. Quant à la Baysère, elle draine les eaux du Laring et de la dépression de Monein depuis les hauteurs d'Estialesq. Comme à Pardies-Pietat, les eaux tombées sur cet important bassin lors des pluies diluviennes se trouvaient concentrées sur PardiesMonein où elles inondaient une grande partie de l'espace occupé aujourd'hui par ce village.

Un même toponyme nomme donc deux sites identiques. Cela me semble exclure le latin parietinae, les «murettes », comme origine. De plus la délimitation des parcelles se faisait naguère par de profonds fossés de drainage, du moins entre le village et les coteaux, et non par des murettes qui auraient retenu l'eau.

La première attestation du village, Bardinae, se trouve dans un texte latin du $11^{\mathrm{e}}$ siècle. Or Bardine ne peut s'être transformé en Pardies entre le $11^{\mathrm{e}}$ et le $14^{\mathrm{e}}$ siècle. Ce nom révèle être en fait la variante basque de Pardies....et l'origine du nom. 
Barda, qui signifie la boue, est un vieux terme pyrénéen que l'on retrouve en basque (cf. Bardos), en gascon (bardissa, bardous) et en espagnol (pardo : «marron »). Ce terme est probablement le même que celui de barthe.

C'est donc "barda », la boue, qui a donné son nom au village, apparemment suffixé par le latin -inas, suffixe à caractère diminutif. Mais la présence en cet endroit d'un suffixe latin est surprenant, de même $q^{\prime}$ 'une appellation « les petites boues ».

Ne peut-on y voir plutôt bard-un-etz, les « lieux boueux », ramené plus tard à Bardinas soit par attraction, soit par suite de la mutation de $u$ en $i$, mutation que l'on rencontre aussi bien en basque qu'en béarnais, cf. Tucoulat et Ticoulat? On aurait donc :

Bardunetz $\rightarrow$ Bardines $\rightarrow$ Pardies, $\rightarrow$ les «lieux boueux ».

NB. - La voie de transhumance dont nous avons vu le rôle à Assat s'est bien gardée de rejoindre la plaine à cet endroit, afin de couper au plus court vers le gué.

Nous pouvons également nous interroger sur la situation respective $\mathrm{d}^{\prime}$ Abos et de St Abit. Ces deux villages se trouvent un kilomètre environ en amont des zones marécageuses des deux Pardies, dans une situation similaire. Or, abstraction faite du préfixe hagionymique St, ils sont construits sur la même racine,-ab-, Abit étant à $A b o s$ ce qu'Esquit est à $E s c o s$, c'est-à-dire quelque chose d'équivalent. Faut-il voir dans «ab » abe, le « fourré », ou le radical d'apotz la « terre humide » ?

Quant au préfixe St, la ferveur populaire le joignit probablement en son temps à Abit, interprété comme Avitus, nom de l'évêque de Vienne du 6e Siècle, à moins qu'il ne s'agisse du radical «send » que l'on retrouve dans Sendets ainsi que dans Sendos (Saint Dos).

\section{Asson}

Prononciation béarnaise : [a'sũ]

Le site est situé à l'extrémité Est du saltus d'entre les gaves de Pau et d'Oloron, à l'endroit où celui-ci vient se coller au premier contrefort des Pyrénées, au centre d'un plateau de $6 \mathrm{~km}^{2}$ environ. On sait qu'au premier millénaire le saltus était sauvage et inhabité.

Comme pour Assat, deux hypothèses possibles :

i) Asson proviendrait de Artzan-un, le «pays des bergers». Ceci correspond assez bien à la situation, car Asson est, pour qui vient de la vallée du gave, le premier village de montagne dont une partie de l'activité est consacrée à l'élevage ovin. Et le plateau était autrefois une vaste lande pastorale. 
ii) Asson proviendrait de Artza-un, le " pays des ours ». Cette dernière hypothèse a ma préférence car la zone est directement ouverte sur l'intérieur montagnard par la gorge de Pédestarres et la vallée de l'Ouzoum, où naguère encore ils pullulaient. Nous trouvons d'ailleurs sur la commune voisine de Lys une maison nommée Latuhe, qui n'est pas répertoriée en 1385 et qui est donc plus récente, dont l'origine se trouve dans la tuhe, trompe rustique de bois utilisée pour la chasse à l'ours au $19^{\text {e }}$ siècle encore. Si Latuhe il y a, c'est qu'habitait là soit un fabriquant de tuhes, soit un habitué des chasses à l'ours.

Enfin, un dicton ne dit-il pas : oursès, barrincoulès, d'Assou, c'est-à-dire : «meneurs d'ours, matraqueurs », d'Asson?

Donc, origine probable : artz/un, le « pays des ours ».

\section{Arros}

Prononciation béarnaise : [ar'ros]

Le village est situé dans la plaine de Licharre, rive gauche, au pied des coteaux. Michel Grosclaude émet de fortes réserves sur une origine « arr / oze », le « lieu des pierres ». C'est qu'il ignorait l'existence sur le territoire de la commune de carrières de pierre, une première, Las Labassères (les « carrières de dalles »), derrière Nay, qui fournissait de grandes dalles grises utilisées dans le pavage des cuisines; une seconde au voisinage de Pardies, au lieu-dit Peyres Blangues, carrière de calcaire fin et blanc, malheureusement gélif, et qui fut exploitée jusqu'au $20^{\mathrm{e}}$ siècle, donnant du travail à la moitié des habitants de Pardies (information issue de la mémoire familiale). C'est de celle-ci que proviendront les pierres qui, sculptées de beaux motifs, orneront au 18 et $19^{\text {e }}$ siècles les façades des grandes fermes que l'on admire en Pays de Nay. D'autres carrières, notamment pour l'extraction des pierres à chaux, existaient sur les flans du Mont du Roi. Il existe enfin sur la commune un chemin dit « des carrières ».

Alors, l'affaire est entendue ? Ce n'est pas certain. Le toponyme existait en effet au tournant du millénaire puisqu'il est attesté "Arrosium » en 1100. Or à cette haute époque, on bâtissait en bois et les sols étaient de terre battue. Et le site internet de la commune situe la mise en exploitation des carrières au $15^{\mathrm{e}}$ siècle. Dans une région où les roches du sous-sol sont occultées par le couvert végétal, la nature rocheuse de celui-ci était-elle suffisamment connue des populations locales pour justifier une telle appellation ? Possible mais pas sûr. L'opinion d'un historien serait ici utile.

Avec quelques réserves donc :

arr/oze, le « lieu de la pierre » 


\section{$>$ LAGOS}

Prononciation en béarnais : [la'gos]

Nous abordons maintenant deux villages, Lagos et Beuste, difficiles à analyser si l'on ne connaît pas l'historique du ruisseau qui les baigne. Michel Groclaude relevait la ressemblance troublante entre Lagos et le Lagoin mais ne pouvait aller au delà. Le Lagoin, qui naît de l'évacuation des eaux pluviales de la forêt de Mourle, réceptacle de superficie très réduite, était condamné à n'être qu'un ruisseau intermittent réduit à des trous d'eau en période de faibles précipitations. Ceci posait un tel problème de salubrité publique pour les hommes et les animaux que le Second Empire détournera en amont de Coarraze une partie des eaux du gave afin de régulariser son débit. Pour nos ancêtres, le Lagoin était donc "le lieu des flaques, des trous d'eau », autrement dit "lago/aïn », qui pouvait se dire aussi «lago/oze ». Le premier sera affecté à nommer le ruisseau et le second le village. Donc :

lago/oze, le « lieu des trous d'eau, des flaques »

\section{$>$ BEUSTE}

Prononciation béarnaise : ['be ${ }^{\mathrm{u}}$ ste]

Très proche voisin du précédent, et en aval de celui-ci, ce village connaissait les mêmes problèmes. Un étymon "beltze », " noir », phonétiquement parlant le plus satisfaisant, n'a ici aucun sens. Peut-être balsta, les mares.

\section{$>$ COARRAZE}

\section{En béarnais : [kwar'razo]}

Le hameau primitif devait être situé sur le promontoire rocheux qui vient, rive droite, surplomber le gave au niveau du pont (l'aspect rocheux se voit parfaitement depuis ce dernier lorsque la falaise est débroussaillée), à l'emplacement du château actuel. Ce promontoire est situé entre la vallée du gave et le vallon du Lagoin. On trouve plus au Nord,

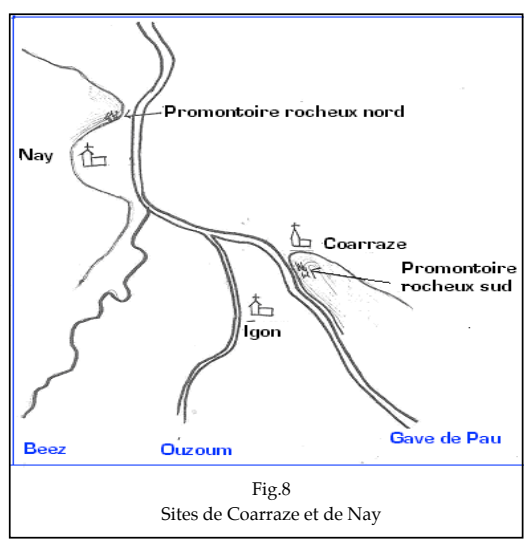
rive gauche, à l'entrée de Nay en provenance de Bourdettes, un deuxième promontoire rocheux dans le quartier de l'Angladure. Je synthétiserai donc le site de Coarraze en trois mots : «promontoire rocheux sud ». 
Mon interprétation
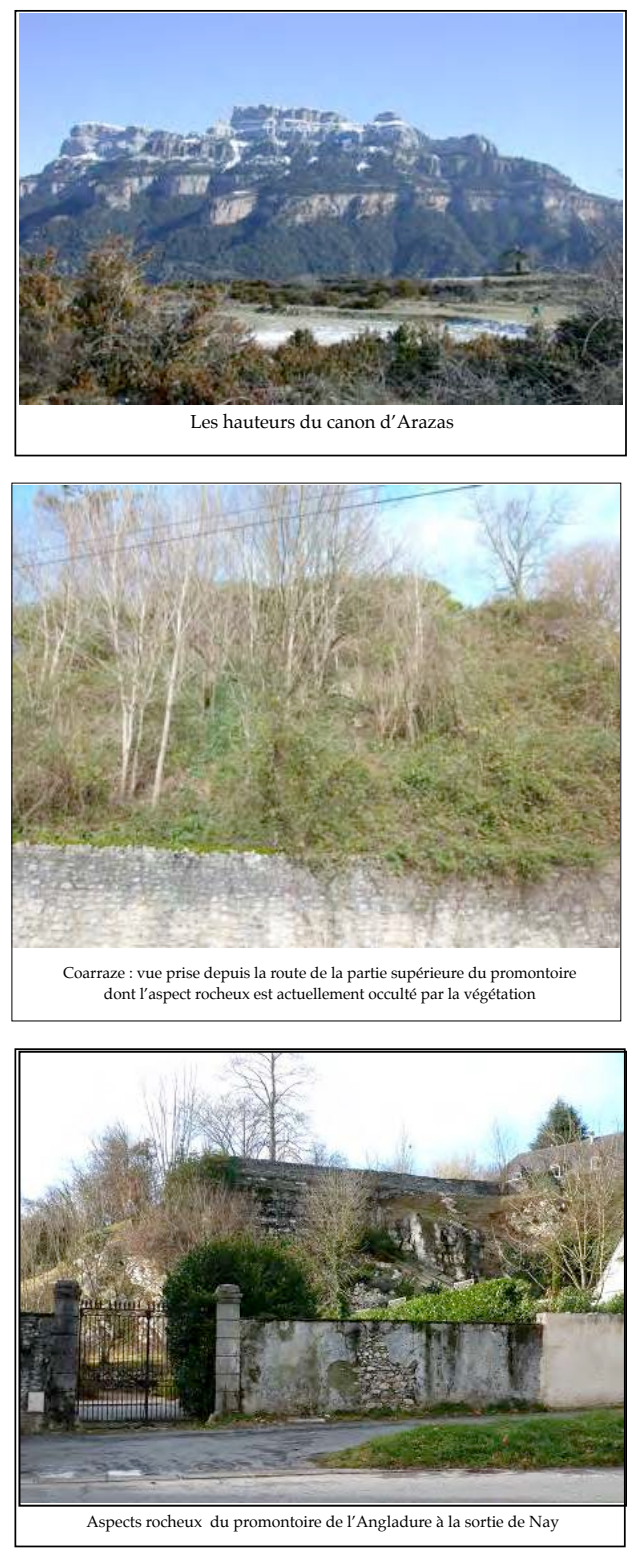

Le village navarrais de Garris, en basque Garusa, est situé sur un promontoire rocheux. L'Itinéraire d'Antonin, au III siècle, le cite sous la forme Carasa qui ressemble étrangement à Coarraze et dans laquelle on reconnaît la racine antique Carr-, « la pierre ». Carasa signifie probablement « barre, piton, promontoire rocheux ».

I 1 s e $\mathrm{m} \mathrm{b}$ le $\mathrm{r}$ a i $\mathrm{t}$ $\mathrm{qu}^{\prime}$ anciennement les deux formes carr- et harr- aient coexisté. Le toponyme Arluzea, la « pierre longue », est attesté en 1025, Carrelucea (Michel Morvan). Nous retrouvons ce terme derrière Gavarnie, avec le cañon d'Arazas (= « des promontoires »?) dans lequel se trouvent plusieurs pitons rocheux dont le plus emblématique est le Tozal del Mallo. On peut donc interpréter Coarraze comme egoarraza, à savoir : le «promontoire rocheux sud ».

L'interprétation « cauda rasa » par un clerc du $12^{\mathrm{e}}$ siècle, parfaite illustration des excès de l'étymologie populaire, révèle que celui-ci ne comprenait plus le basque, soit que cette langue ait à l'époque disparu de la rive droite du gave, soit que le rédacteur soit extérieur au Pays de Nay. 


\section{> GUATARAM (Betharram)}

Prononciation béarnaise : [gwata'ram]

Au niveau de ce lieu-dit, le gave vient buter sur l'abrupte falaise rocheuse, ménageant un étroit passage vers l'amont, en direction de la toute proche frontière de la Bigorre. Très tôt, afin de fermer ce passage et d'empêcher toute invasion en provenance de celle-ci, un sanctuaire marial sera construit à cet endroit, sécurité renforcée en 1335 par la création, à proximité immédiate, de la

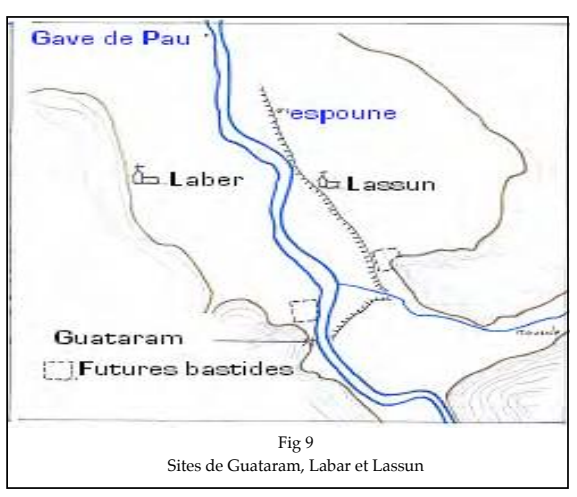
bastide de Lestelle. Nous avons

là, rive gauche, la porte d'entrée de la plaine de Nay, c'est-à-dire du Béarn.

Le site peut donc être résumé par : « la porte sud de la vallée » ou « le passage au sud de la vallée ", ce que traduit parfaitement ego-ate-aran. Le basque actuel dirait plutôt aran/ego/ate, mais l'inversion déterminant/ déterminé par rapport à celui-ci était courante dans le basque de Béarn, ou de Soule, probablement sous l'influence du roman. Comparer Etchebarne et Barneix. À coup sûr :

egoataran, la « porte » ou le « passage au sud de la vallée».

\section{$>$ LASSUN}

Il était situé à un kilomètre au nord de Montaut, sur le rebord de l'espoune, à proximité de l'endroit où le gave vient lécher cette dernière. On pense à latz/une, le ruisseau, mais nul ru ne se trouve dans les parages. Par contre le gave, qui au sortir de la montagne, ralentit sa course dans une série de méandres, ne pouvait qu'abandonner force gravier dans cette zone. Je suggère donc de voir dans Lassun leia/tz/un, la " gravière ». On rapprochera de Lassun le toponyme Laas, village qui occupe dans la vallée $\mathrm{du}$ gave d'Oloron une position très semblable en bordure du gave. Au résumé :

leia-tz-un, le lieu où le sable abonde

\section{$>$ LABER}

Michel Grosclaude dit que ce village se trouvait assez loin du site de Lestelle. Il se trouvait donc plus au nord, vers Igon, à proximité et en contrebas du plateau d'Asson qui occupe l'extrémité sud-est du saltus. Il est plus que probable qu'au premier millénaire ce plateau n'était qu'une 
lande pastorale dominée par l'oppidum. On peut donc interpréter Laber comme ala-behere, " en bas des pâturages ». Soit donc :

alaber, en bas des pâturages

\section{BOEIL}

Prononciation, en béarnais : [bwei]

Nous allons maintenant examiner le cas de trois toponymes très anciens si, d'une part, l'on considère leur morphologie, et d'autre part l'emplacement des hameaux sur des sites défensifs. Il s'agit de Boeil, Baudreix et Baliros. Ils ont pour point commun d'occuper sur l'une ou l'autre des deux « espounes » encadrant le gave une singularité de celle-ci facilitant la défense. Rappelons qu'au début du premier millénaire ce que nous appelons en

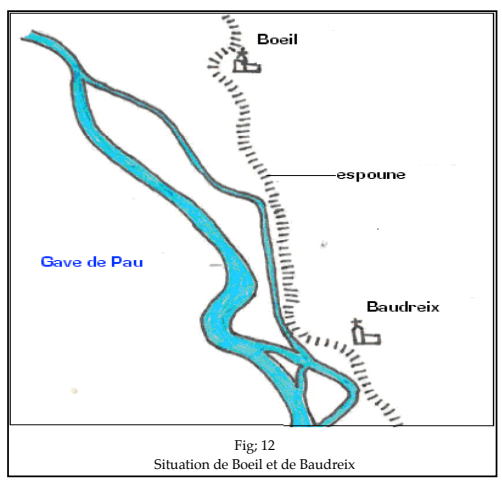
béarnais « espoune » se disait «bal » ou «buka ».

Le village de Boeil est situé sur le rebord de l' "espoune ", à $2 \mathrm{~km}$ de Baudreix. Celle-ci fait ici une baïonnette qui ménage une position défensive favorable, occupée à l'origine par le hameau primitif et

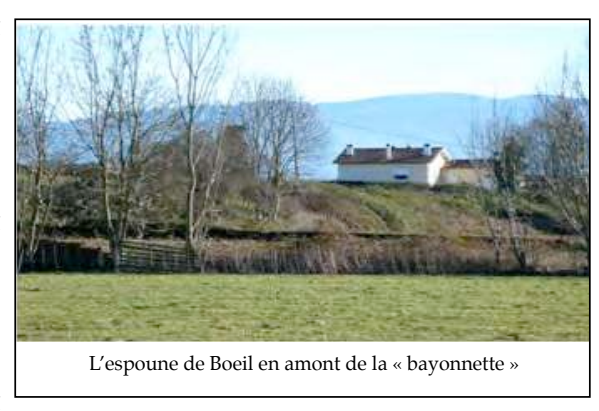
aujourd'hui par l'église, le cimetière et une maison.

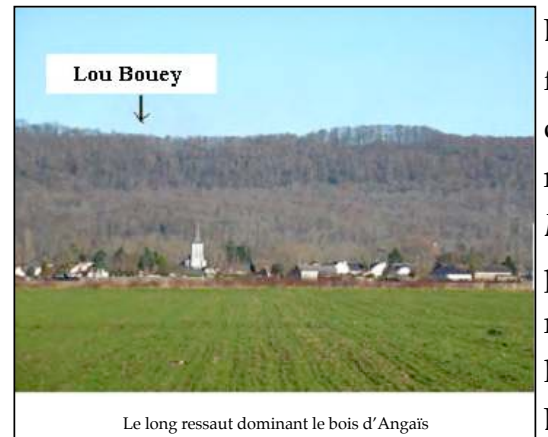

Boeil implique par ailleurs une forme ancienne buk'le qui a convaincu Michel Grosclaude que nous avions là un andronyme latin Boviculus, le «bouvillon ». Ceci est phonétiquement parlant possible, mais fortement improbable, car tous les villages de ce type, à savoir en Bigorre Bouilh-Davant et BouilhPerulh, et en Béarn Boueilh, Bouelho et Bouillon, sont situés sur le rebord d'un haut talus, de même que le lieu dit Lou Bouey, (qui n'est pas un hameau), sur l'abrupt dominant la colline 
voisine d'Angaïs, ainsi qu'un second "Lou Bouey », au pied de l'espoune d'Artigueloutan.

Je pense qu'il faut donc voir dans la racine $b u k^{\prime}$ le basco-aquitain buka, " la hauteur longitudinale, le haut talus », dont héritera le basque moderne muga.

$B u k$-ili, le «village du rebord ", est à mon avis erroné, car les lieux-dits Lou Bouey (sur la commune d'Angaïs et celle d'Artigueloutan) ne sont pas des sites habités. Mouguère, correspondant basque de Boeilh, nous oriente plutôt vers buk-ele, le terme « ele » restant pour l'instant obscur. Orpustan, qui en ignore le sens, signale qu'il est attesté pour suffixer des mots tels que larre, etxe, zubi, hegi, ezki, etc. Pour Luchaire, il signifiait : " le lieu de $» . .$.

- Mouguère, également située sur le rebord d'une hauteur, est interprétée Muga-Ili, la « ville de la hauteur ». Mais Mugili aurait plutôt donné Mugiri. Il est probable que Mouguère a la même origine que Boeil, à savoir bukele, cet étymon aboutissant en basque à mugere. Je propose donc, pour Mouguère et Boeil :

bukeke, le « lieu du haut talus, de l'abrupt ».

\section{$>$ BAUDREIX}

Prononciation béarnaise : [ba $\left.{ }^{u^{\prime}} \mathrm{dr} \varepsilon f\right]$

Singularités du site :

Le Baniu, ancien lit majeur du gave, aujourd'hui secondaire, vient mordre le talus ou " espoune », alors qu'ailleurs il en est éloigné. Mais le lit du gave est changeant.

Le talus possède là la plus haute élévation de la plaine $(15 \mathrm{~m})$ et surtout un profil en forme de corne, alors qu'il est en général plus ou moins rectiligne. Cependant le village, qui ne pouvait à l'origine se trouver en bas de l'espoune, a été détruit vers le 17 e siècle par une crue du gave venue probablement saper l'obstacle que représentait la corne qui sera en partie emportée ainsi que les habitations situées au-dessus, forme en corne qui subsistera néanmoins après le recul consécutif à la crue.

Mon opinion :

" Bawdrèch ", qui implique une forme ancienne Baldareix ou Baladaretche s'explique par «Bal-Adar-Etxe » dont les trois termes sont courants en toponymie basco-aquitaine : bal, la « hauteur, le talus », adar la « corne », et etxe le « hameau ». Soit donc:

bal-adar-etxe, le « hameau de la corne du talus » . 


\section{BALIROS}

Prononciation en béarnais : [bali'ros]

Le village est situé rive gauche du gave sur un promontoire non rocheux formé par l'espoune d'une part et, d'autre part, la dépression creusée par la réunion du Luz et du Gest lors de son franchissement de celle-ci (voir carte). Le promontoire ainsi formé domine la dépression et la terrasse basse du gave d'une dizaine de mètres ménageant une intéressante position défensive en forme de dentelure de fougère et verrouillant le passage vers Assat. Un tel site est probablement d'occupation très ancienne, antérieure à la conquête.

Il est important de noter que Baliracq, dans le Vic Bilh, possède exactement la même situation : sur un éperon non rocheux similaire au précédent, formé par la vallée du grand Lèes d'une part et le ravin d'un ruisseau de l'autre. Mais ici la hauteur domine la partie basse de 80 mètres.

Il est dans ces conditions impossible d'attribuer une étymologie Valerius, mais bal-ir-oz, dans lequel bal est la « hauteur, le talus », et -ir- un terme obscur relatif au site. Le suffixe $-a c q$ ne doit pas surprendre en Vic Bilh. Peut-on voir dans ira une allusion à la ressemblance qu'ont les deux éperons avec les dentelures de la feuille de fougère ?

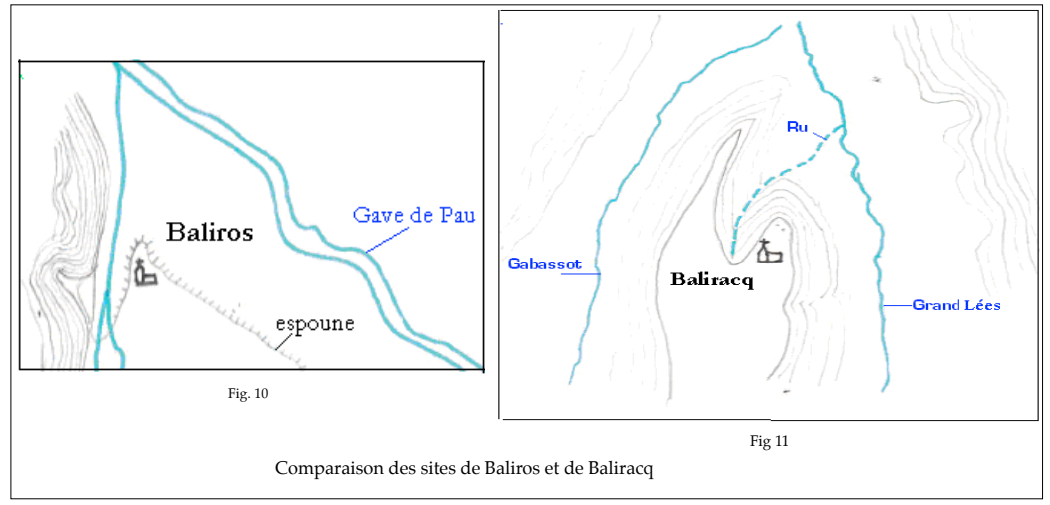

Proposition : BAL-IR-OZE, la « feuille de fougère de l'espoune. Autrement dit le « promontoire en feuille de fougère ».

\section{IGON}

Prononciation béarnaise : [i'goun

Michel Grosclaude propose comme étymologie ihigune, le « lieu des joncs ». Ceci est fort probable, car le site se trouve au confluent de l'Ouzoum et du gave. D'accord, donc, pour :

ihigune, la jonchaie 


\section{$>$ NAY}

Prononciation en béarnais : [nai]

Il propose également pour Nay, nagi, le « bas-fond humide ». D'accord ici aussi avec son analyse :

nagi, le « bas-fond humide »

\section{$>$ ARTHÈZ}

Prononciation en béarnais : [ar'tes]

Je le suivrai ici encore :

artetz, les « chênes verts, les broussailles ».

\section{> MIREPEIX}

Prononciation béarnaise : [mira'pef]

Ce village est situé sur le rebord de $\mathrm{l}^{\prime}$ " espoune », fort proche à cet endroit du gave, face à un magnifique panorama des montagnes, et à l'aval immédiat de l'ancienne commune de Clarac absorbée au 19e siècle par Nay. Michel Grosclaude nous a présenté dans l'ouvrage cité en référence les deux hypothèses en concurrence, toutes deux romanes, et toutes deux en cohérence avec le site.

- Miră/peix, regarde les montagnes.

Cette hypothèse est rendue plausible par l'existence de Mirepoix en Ariège. Mais le latin «podium » a donné en Béarn «pouy », " poey » et non « pech », courant quant à lui dans le Toulousain et l'Aude. Sorti aujourd'hui de l'usage, il y est interprété parfois comme " poisson » par les populations locales. D'autre part dans la plaine de Nay une appellation « regarde la montagne » ne serait absolument pas discriminante, car la vue sur les Pyrénées y est fort belle depuis de multiples endroits.

- Miră/peix, regarde les poissons.

Une telle interprétation ne fait pas de doutes pour l'île de Mirepech située dans l'Adour à Ste Marie de Gosse. Mais quand on connaît la propension de ce fleuve à s'ensabler, on peut douter de l'ancienneté de l'appellation. Et notre Mirepeix resterait de toutes façons le seul village à porter un nom « regarde les poissons ».

Comme précédemment, l'appellation ne serait absolument pas discriminante, car d'autres villages de la plaine de Nay sont situés au bord de l'eau.

On peut imaginer une troisième hypothèse romane car le Palay donne pech signifiant " pâturage » dans le Lavedan voisin. Mais, outre qu'ici encore il n'y aurait pas discrimination, on est en droit de penser que le 
clerc latiniste du $13^{e}$ siècle aurait transcrit ce nom Mirapascuum et non Mirapisces.

Par ailleurs, Mirepeix appartient à la dizaine de toponymes suffixés en -eix, prononcé en gascon -èch, tels Baudreix, Azereix, Tarasteix, Orleix, Sireix, Baleix, ainsi que, graphiés différemment : Omex, Artouech, etc. Tous sont situés sur un espace restreint à la limite entre le Béarn et le Bigorre, et totalement absents ailleurs. Ces toponymes ne sont pas romans et seul ici Sireix rappelle peut-être Rome : le hameau de Sirus ? Ce dernier point milite fortement pour une origine non romane.

Peut-on dans ces conditions rechercher du côté aquitain?

Nous venons de voir que ce village est situé à l'aval immédiat de Clarac, tous deux au bord du gave. Or, qui dit Clarac, dit le domaine de Clarus, c'est-à-dire qu'une villa aquitano-romaine existait probablement à cet endroit, singularité combien marquante dans le paysage. Or on peut voir dans -peix les termes - pe/etche (cf. Goyeneix < Goyen/ etche), à savoir « les maisons, le hameau à l'aval de.. ». Mira est-il alors susceptible de procéder de Villa?

À supposer que les Aquitains locaux aient eu l'intention de nommer cet habitat « le hameau à l'aval de la villa ", ils devaient obligatoirement emprunter le terme «villa » et l'adapter à la phonétique de leur langue. Celle-ci laisse dans un premier temps intacte la géminée -1l- qui ne sera réduite à -1- qu'à la fin du premier millénaire. En ce qui concerne le « v » initial, il est intégré dans les emprunts sous la forme de «b $»$ ou de « $\mathrm{m}$ », surtout à l'initiale. Luis Michelena écrit dans Fonética Històrica Vasca (13.6., p.268) : « Pero ademàs vasc. $m$ - responde a menudo a lat. $b$-, v-, y...etc. ». Il donne comme exemples :

— mimen < lat. vimen, osier

— makila < lat. bacillum, bâton

Il ne précise pas, sauf erreur de ma part, si cette mutation est contemporaine de l'emprunt, mais il cite l'explication suggérée par Martinet qui ne l'exclut pas : «...Dentro de la hipòtesis de que el fonema vasco / b / no era aùn plenamente sonoro en la época de los primeros contactos con el latìn, se comprende muy bien que los vascos hubieran reproducido en ocasiones con su fonema labial sonoro / mb/ las labialas sonoras latinas b y v. »

Dans cette hypothèse, l'emprunt aurait pu se faire sous la forme milla. Le toponyme millapetche, passé dans le bas latin local, serait devenu vers le 5 ou $6^{\text {e }}$ siècle mirapetche conformément à la phonétique du proto-gascon, puis au $7^{\text {e }}$ siècle mirapech à la chute des voyelles terminales. D'autres variantes sont possibles. 
Dans le cas où il serait avéré que la mutation basque $\mathrm{v}-\rightarrow \mathrm{m}$ - serait tardive et daterait du bas Moyen-âge, cette hypothèse ne tiendrait bien sûr plus et le problème étymologique de Mirepeix resterait entier. J.B. Orpustan, spécialiste de la toponymie basque interrogé sur ce point, estime cette hypothèse très peu viable, ville/bila n'étant pas attesté dans les citations médiévales anciennes.

Avec donc de sérieuses réserves

Milla/pe/etche = le hameau à l'aval de la villa

qui n'est somme toute pas plus saugrenue que les précédentes et a l'avantage d'être la seule discriminante, conforme au site, en même temps qu'à l'esprit prosaïque de nos ancêtres.

\section{VI) Conclusion}

Résumons-nous :

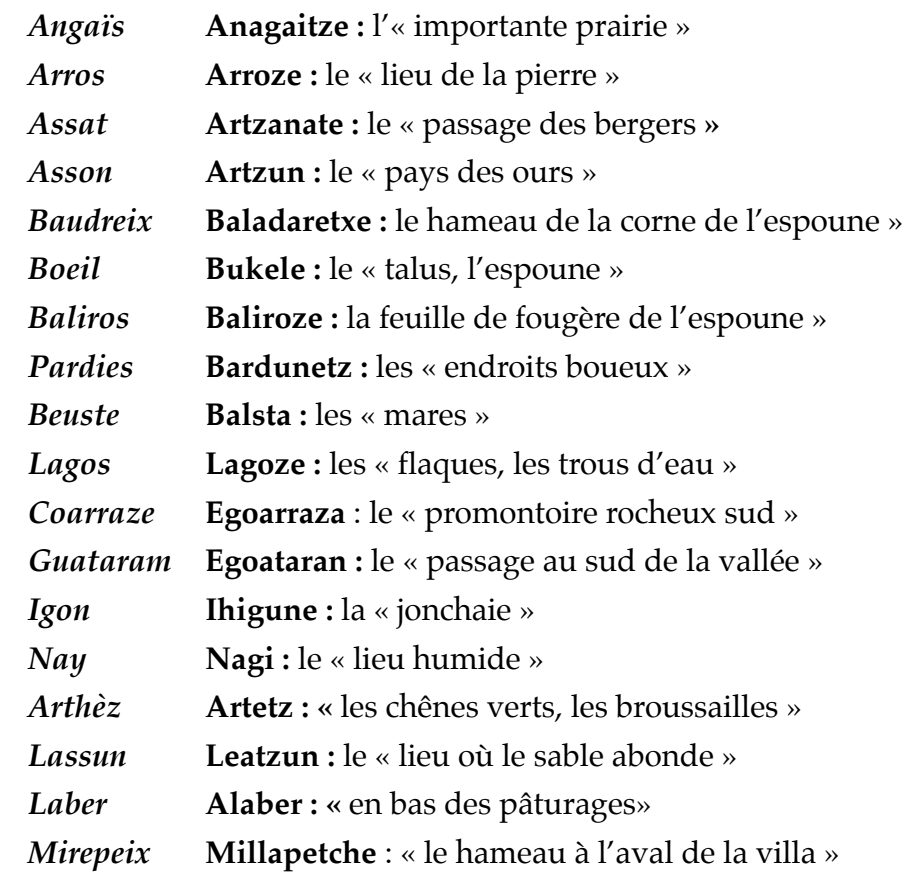

Comme on le voit, en ces temps reculés nos ancêtres repéraient les sites par leur singularité et leurs descendants romanisés continueront de même pour nommer les quartiers de leurs villages, soit qu'ils conservent l'ancien micro-toponyme, soit qu'ils en créent de nouveaux dans leur nouvelle langue.

L'étymologie d'un toponyme est une chose, sa morphologie à un instant donné en est une autre. Car, rappelons-le, les langues évoluent. La 
morphologie de ceux de la précédente liste est différente de celle qu'ils possédaient lors de la disparition du basque de la plaine de Nay. Il s'agit de la forme qui était la leur lorsqu'ils passèrent dans la langue romane locale, et la date de ce passage est différente pour chacun d'eux. L'image de cette série est en fait similaire à celle du ciel où ce que l'on voit ne correspond pas à la réalité présente. Il est fort possible que Baudreix et Baliros par exemple, villages très anciens, aient eu à l'époque de la débasquisation les formes « Maradareix » et « Mariroz », ainsi que Boeil la forme « Mugere».

Coarraze par contre devait être plus récent et son nom avoir conservé dans l'esprit des locuteurs un caractère descriptif, car nous avons Egoarraza et non Egocarraza.

Une dernière observation concernant la série reconstituée : la morphologie des toponymes nous donne une certaine indication sur leur ancienneté, qui n'est pas forcément la même que celle des villages qui s'établiront sur ces sites. Ainsi le suffixe «-oz » étant le plus ancien, il en est probablement de même de Lagos et Baliros. On peut également penser que les sites favorables à la défense ont été occupés parmi les premiers. D'autre part, les racines buka et bal sont archaïques ; elles évolueront vers muga et bar. Bal se retrouvera sous cette forme dans Barzun (bar/tzu/un, le lieu des talus, ou limitrophe), bar ainsi que muga ayant naturellement évolué vers le sens de limite. Ce village est en effet établi au pied du long talus rectiligne et élevé séparant la vallée de l'Ousse du plateau de Ger. Ce talus, situé rive droite, domine celle-ci d'une centaine de mètres. Rive gauche, deux ou trois talus d'une dizaine de mètres de haut la séparent de la terrasse supérieure où se trouve la route Pau-Lourdes. Il s'agit bien ici $\mathrm{du}$ lieu des talus. On retrouve « bal » sous la forme «bar » dans Barlest (bar/l/etz) de situation et de sens similaires, le létant consonne de liaison comme dans «Behor-l-egi », la demeure des juments. Ces deux toponymes sont donc soit plus récents, soit passés dans le roman local plus récemment.

On peut, pour ces différentes raisons, estimer que Boeil, Baliros, Lagos et Baudreix comptant parmi les plus anciens. Par contre, Coarraze et Guataram sont peut-être les plus tardifs, bien que le site de Coarraze soit particulièrement favorable à la défense. Seule l'archéologie pourrait ici trancher.

En ce qui concerne Pardies et Assat, ces deux noms ont vu dans leur variante romane la chute de leur «-n-» intervocalique, ils remontent donc au moins au 6 ou 7 e siècle, si du moins nous ne nous sommes pas trompés dans leur étymologie. 
La toponymie de la région de Nay est en majorité proto-basque ou basque. Ceci ne saurait être représentatif de l'ensemble du Béarn, mais nous laisse imaginer que la toponymie de celui-ci est moins latine qu'on ne pense.

\section{Bibliographie}

Dauzat, Albert \& RostAING, Charles (1963) Dictionnaire des noms de lieux de France, Cumer-Fantin, Saint-Etienne.

Groclaude Michel (1991), Dictionnaire toponymique des communes du Béarn, Pau, Escòla Gaston Febus, 1991. (DTCB).

Fénié, B. \& J.J. (1992), Toponymie gasconne, S.O, Éditions Sud-Ouest.

Michelena, Luis (1990) Fonética Històrica Vasca, Bilbao.

Morvan, M. (2004), Noms de lieux du Pays Basque et de Gascogne, Christine Bonneton.

Orpustan, J.B. (1984), Les noms de maisons médiévales basques, thèse.

Orpustan, J.B. (1999), La langue basque au Moyen-Age, IXe-XVe siècles, Izpegi.

Oyhamburu, P. (1991), Euskal deituren histegia, Dictionnaire des patronymes basques, Éditions Hitzak.

Rostaing, Charles (1965), Les noms de lieux, "Que Sais-je », Presses Universitaire de France.

Collectif (1996), Archéologie en Béarn, Actes du colloque d'Arzacq. Université de Pau, Segur.

(1385) Recensement des feux de Béarn, 